\section{FDA Expands Requirements for Tissue Product Safety}

The FDA issued a final rule January 19, 2001, requiring establishments that manufacture human cellular or tissue-based products to register their business and list their products with the FDA. The rule is part of a series of three rules proposed as a result of the agency's plan to improve regulation of such products. The other two rules, which have not been finalized, are a proposed rule for donor-suitability requirements and a good tissue practice (GTP) regulation proposed in early January 2001.

Establishments that recover, screen, test, process, store, or distribute human tissue intended for transplantation must begin complying with the rule April 4, 2001. The effective date for all other human cells, tissues, and cellular and tissue-based products is January 19, 2003. The FDA expects the other two proposals to be finalized by then, the rule says.

The proposed regulation on current GTP (GTP covers the methods, facilities, and controls used for the manufacture of all human cellular and tissue-based products and includes adequate organizational structure and sufficient personnel, standard operating procedures for all significant steps in manufacturing, control and validation of manufacturing processes, maintenance of a complaint file, and procedures for tracking the product from donor to recipient and from recipient to donor).

Also under the proposed GTP rule, manufacturers would be required to report adverse reactions and certain product deviations, to have adequate labeling, and to allow FDA inspections. Certain cellular and tissue-based products that require licensing or premarket approval as biological products or medical devices would be subject to more comprehensive requirements based on their risks, the FDA says.

FROM: Food and Drug Administration. Human cells, tissues, and cellular and tissue-based products. Establishment registration and listing. Final rule. Federal Register January 19, 2001;66(13):5447-5469. http://www.fda.gov.

\section{JCAHO to Assess Effectiveness of Staffing}

On January 11, 2001, the Joint Commission on Accreditation of Healthcare Organizations (JCAHO) announced plans to develop a new approach to assessing the effectiveness of staffing in healthcare organizations nationwide. The new process will use performance indicators to screen for potential staffing issues and will be pilot tested during 2001.

The effort is part of JCAHO's continuing commitment to identify and address potential opportunities to improve patient-care quality and patient safety through its accreditation process. Effective staffing has been identified as a current issue of significant concern among healthcare professionals and the public.

The new assessment initiative will draw upon both human resources and clinical outcome measures. Human resources measures will encompass all staff that provide healthcare services, including direct patient caregivers, such as registered nurses and respiratory therapists, as well as clinical-support professionals responsible for pharmacy, laboratory, and radiology services. The approach is designed to emphasize the relation between human resources and clinical outcomes and recognizes that no single measure can reliably describe staffing effectiveness.

Current JCAHO standards require accredited healthcare organizations to determine and provide the right number of qualified and competent staff to meet the needs of patients. These determinations are usually based on internal formulae that reflect numbers of patients and how sick they are.

FROM: JCAHO. Joint Commission to develop a new approach to assessing the effectiveness of staffing in healthcare organizations. www.jcaho.org/news.frm.html.

\section{GAO Report: Needlestick Prevention Devices Are Cost Effective for High-Risk Exposures}

In response to a request by Congress to examine the potential cost benefit of needlestick prevention devices, on November 17, 2000, the US General Accounting Office (GAO) reported that needlestick prevention devices are cost-effective. The report showed the cost-effectiveness for postexposure treatment of a moderate- to high-risk exposure if the increased cost of the safety device was medium ( 2.0 times more costly) to low ( 1.5 times more costly), compared to a conventional device.

GAO assumes postexposure treatment (ie, tests and treatment for exposure to bloodborne pathogens) costs from $\$ 500$ to $\$ 2,500$, and estimates that eliminating 69,000 needlesticks per year would reduce postexposure treatment costs for injured healthcare workers in hospitals by between $\$ 37$ million and $\$ 173$ million per year.

They also note that, while only a subset of healthcare workers who suffer needlestick injuries subsequently become infected, adoption of needles with safety features also may reduce costs associated with longer term treatment for those workers. For example, the average annual cost of treating a person with HIV has been estimated at between $\$ 20,000$ and $\$ 24,700$ in 1996 .

FROM: General Accounting Office. Occupational safety: selected cost and benefit implications of needlestick prevention devices for hospitals. http://www.gao.gov/new. items/d0160r.pdf.

\section{OSHA Revises Bloodborne Pathogen Standard-Mandates Sharps Safety Devices}

On January 18,2001 , OSHA published a revision to the Bloodborne Pathogen Standard, to comply with the mandate of the Needlestick Safety and Prevention Act signed by 
Congress in November 2000 mandating OSHA to enforce the use of sharps safety devices. The effective date of the revised regulation is April 18, 2001. States with their own occupational safety plans have 6 months to adopt a comparable standard. If a state has a stricter needle safety law already in place, the state law will still apply. The only hospitals not covered under the rule are public hospitals in states without their own occupational health and safety agency.

The revisions include a new definition of an engineering control to include as examples, a device with engineering sharps-injury protection, and a needleless IV device. In addition, there must be an annual review and revision of the exposure control plan to include consideration appropriate, commercially available, and effective safer medical devices to reduce risk of exposures. An appropriate safer medical device is one that would not jeopardize patient or employee safety or be medically contraindicated, the rule states. The plan must include a description of the devices, methods used to evaluate them, and reasons for adoption. Employers are also required to include frontline workers in the identification, selection, and evaluation of these devices, and to document this input in the plan. Also, the revised rule requires a sharps-injury log that collects information on the type and brand of device involved in the incident, where the incident occurred, and an explanation of the incident with adequate privacy protection.

OSHA also referred to the publication of OSHA's final recordkeeping rule, which was published separately. This revised recordkeeping rule introduced new forms for recording occupational injuries and illnesses, but noted that the detailed sharps-injury log must be kept in accordance with the revised Bloodborne Pathogen Standard.

OSHA has commented that this revised rule does not change the enforcement of the use of sharps safety devices, which were already clarified in the revised November 1999 Enforcement Procedures for the Bloodborne Pathogen Standard. The January 18, 2001, revision only formalizes this. OSHA has been citing hospitals for lack of use of sharps safety devices since November 1999 . There has been some flexibility in the citations issued for employers that have evidence of adoption of some devices and a detailed plan outlining the plan for completion with timelines.

FROM: Department of Labor. OSHA. Occupational exposure to bloodborne pathogens: needlestick and other sharps injuries. Final rule. Federal Register 2001;66:53175325.

\section{JCAHO Approves New Patient Safety Standards}

The Joint Commission on Accreditation of Healthcare Organizations (JCAHO) has approved standards directly focused on patient safety and medical error reduction in hospitals. The implementation date for the standards is expected to be July 2001. The new standards expand on current JCAHO standards, which require healthcare organizations to identify, report internally, and analyze sentinel events and to take action to prevent their recurrence. Requirements for patient-safety programs will be added in the following areas:

Leadership: Hospital leaders are to encourage error identification and remedial steps to prevent future errors. Individual blame or retribution should be minimized for those involved in an error or in reporting an error.

Improving organization performance: Hospitals are to implement a program for proactive assessment of high-risk activities related to patient safety and to undertake appropriate improvements. The hospital will select which activities to assess based on available knowledge, including information that is provided by JCAHO through its study of adverse events that seriously harm patients (sentinel events).

Information management: Patient-safety-related data should be aggregated by the hospital to identify risk to patients, and results should be communicated effectively to caregivers and others involved in patient safety to reduce these risks.

Other functions: Patient safety should be emphasized in areas such as patient rights, education of patients and their families, continuity of care, and human resources management. The patient or the patient's family should be informed about the results of care, including unanticipated outcomes.

FROM: icanNEWS. January 2, 2001. http://www. icanprevent.com.

\section{US Action Plan to Combat Antimicrobial Resistance}

An action plan, developed by an interdepartmental task force, was unveiled recently that provides the United States with a comprehensive approach to combat antimicrobial resistance. The plan designates priorities and identifies responsible agencies and timelines.

The CDC, the NIH, and the FDA led a task force of 10 agencies and departments that included the Agency for Healthcare Research and Quality, the Health Care Financing Administration, and the Health Resources and Services Administration at HHS, as well as representatives from the Department of Agriculture, the Department of Defense, the Department of Veterans Affairs, and the EPA.

The plan has four major components: surveillance, prevention and control, research, and product development. Top priorities of the four major sections include:

Surveillance. The CDC will work with state health departments and other task force members to design and implement a plan that will define national, regional, state, and local antimicrobial-resistance surveillance responsibilities, so that these entities are coordinated and use similar methodology. Additionally, systems will be developed that can monitor patterns of antimicrobial-drug use in human medicine, in agriculture, and in consumer products.

Prevention and control. A national public education campaign will be launched to reduce the overuse and 